\title{
A NOTE ON COMPACT KÄHLER-RICCI FLOW WITH POSITIVE BISECTIONAL CURVATURE
}

\author{
Huai-Dong Cao and Meng Zhu
}

\begin{abstract}
We show that for any solution $g_{i \bar{j}}(t)$ to the Kähler-Ricci flow with positive bisectional curvature $R_{i \bar{i} \bar{j} \bar{j}}(t)>0$ on a compact Kähler manifold $M^{n}$, the bisectional curvature has a uniform positive lower bound $R_{i \bar{i} j \bar{j}}(t)>C>0$. As a consequence, $g_{i \bar{j}}(t)$ converges exponentially fast in $C^{\infty}$ to a Kähler-Einstein metric with positive bisectional curvature as $t \rightarrow \infty$, provided we assume that the Futaki-invariant of $M^{n}$ is zero. This improves a result of D. Phong, J. Song, J. Sturm and B. Weinkove [22] in which they assumed the stronger condition that the Mabuchi K-energy is bounded from below.
\end{abstract}

\section{The Results}

We consider solutions $g_{i \bar{j}}(t)$ to the normalized Kähler-Ricci flow

$$
\frac{\partial g_{i \bar{j}}}{\partial t}=-R_{i \bar{j}}+g_{i \bar{j}}
$$

with positive holomorphic bisectional curvature $R_{i \bar{i} \bar{j} \bar{j}}(t)>0$ on a compact Kähler manifold $M^{n}$. Our main result is a uniform lower bound on the bisectional curvature of $g_{i \bar{j}}(t)$.

Theorem 1.1. Let $g_{i \bar{j}}(t), 0 \leq t<\infty$, be a solution to the normalized Kähler-Ricci flow (1.1) with positive holomorphic bisectional curvature $R_{i \bar{i} \bar{j}}(t)>0$ on a compact Kähler manifold $M^{n}$. Then, there exists a positive constant $C_{1}>0$ such that

$$
R_{i \bar{i} j \bar{j}}(t)>C_{1}
$$

for all $0 \leq t<\infty$.

In the Riemannian case, powerful curvature pinching estimates were first proved by Hamilton for Ricci flow on compact 3-manifolds with positive Ricci curvature [13] and on 4-manifolds with positive curvature operator [14]. More recently, curvature pinching estimates were obtained by Böhm-Wilking [5] for Ricci flow on compact $n$-manifolds $(n \geq 5)$ with positive curvature operator (see also Brendle-Schoen [7] with a certain positive curvature condition that is weaker than $1 / 4$-pinch). These pinching results are proved by using Hamilton's advanced maximum principle, and a key step is to show that the pinching holds under the reaction ODE system of the evolution equation of the curvature tensor. However, the Kähler case appears to be more subtle since the reaction ODE system of the curvature tensor doesn't even preserve the lower bound of the bisectional curvature, even in complex dimension 2.

Received by the editors March 23, 2009.

${ }^{1}$ Research partially supported by NSF grants DMS-0354621 and DMS-0506084. 
In fact, Hamilton [17] showed that under the reaction ODE system, there could be a solution that emerges from the curvature operator matrix of $\mathbb{C P}^{2}$ and approaches to the curvature matrix of $\mathbb{C} P^{1} \times \mathbb{C}$. Of course this doesn't mean it actually happens to the curvature evolution $\mathrm{PDE}$ system, rather a different argument from the Riemannian case is needed in order to prove the curvature pinching in the Kähler case. In this paper we use a compactness argument to prove a positive uniform lower bound for the evolving bisectional curvature. The proof relies on a result of H.-L. Gu [12] (see Lemma 2.1 below) which in turn depends on the strong maximum principle developed by Brendle-Schoen [6] for certain degenerate parabolic operators. We also use a lemma from Chen-Sun-Tian [10]. While we are able to prove a uniform positive lower bound on the bisectional curvature, it remains very interesting to see if one can show the pinching of holomorphic sectional or bisectional curvature.

Based on Theorem 1.1 and the work of Phong-Song-Sturm-Weinkove [22], we obtain the following

Theorem 1.2. Let $\left(M^{n}, g_{0}\right)$ be a compact Kähler manifold with positive bisectional curvature. Assume that the Futaki invariant of $M^{n}$ is zero. Then the solution $g_{i \bar{j}}(t)$ to (1.1) with initial metric $g_{0}$ converges in $C^{\infty}$-norm exponentially fast to a KählerEinstein metric $\tilde{g}$. In particular, $M^{n}$ is biholomorphic to the complex projective space $\mathbb{C} P^{n}$ and $\tilde{g}$ is the Fubini-Study metric.

Theorem 1.2 improves one of the main results of [22], in which it is assumed that the Mabuchi $K$-energy is bounded below. As pointed out in [22], the vanishing of the Futaki invariant is, at least a priori, much weaker than the K-energy being bounded below. Of course, one would like to drop the assumption on Futaki invariant as well in order to get a Ricci flow proof of the Frankel conjecture without relying on the works of Mori [20] and Siu-Yau [26].

Remark 1.1. For $n=1$, Hamilton [15] showed that under the Ricci flow, one can deform any metric of positive Gauss curvature on a compact surface $\Sigma$ with positive Euler number to a metric of constant positive Gauss curvature. Later in [11], Ben Chow showed that any initial metric on $\Sigma$ becomes positive curved after some finite time, hence converges to a metric of constant positive Gaussian curvature. For $n=2$, Theorem 1.2 was derived in [22] without using Theorem 1.1 due to the basic fact that in complex dimension 2 nonnegative bisectional curvature implies nonnegative curvature operator on (2,0)-tensors (also see, e.g., [27]).

\section{The Proofs}

Proof of Theorem 1.1. Let $g_{i \bar{j}}(t), 0 \leq t<\infty$, be a solution to the normalized Kähler-Ricci flow (1.1) on a compact $M^{n}$ with positive bisectional curvature.

Claim: There exists some positive constant $C>0$, such that

$$
R_{i \bar{j}}(t)>C g_{i \bar{j}}(t)
$$

for all $t \geq 0$. 
We argue by contradiction. Suppose that the claim is not true. Then we can find a sequence of positive number $\epsilon_{k} \rightarrow 0$, and a sequence of points $\left\{\left(x_{k}, t_{k}\right)\right\}_{k=1}^{\infty}$ in space-time with $x_{k} \in M$ and $t_{k} \rightarrow \infty$ as $k \rightarrow \infty$ such that

$$
\min _{1 \leq i \leq n} R_{i \bar{i}}\left(x_{k}, t_{k}\right) \leq \epsilon_{k}
$$

Now we can choose a unitary frame $E^{k}=\left\{e_{1}^{k}, \cdots, e_{n}^{k}\right\}$ at the point $x_{k}$ and the time $t_{k}$ so that

$$
R_{1 \overline{1}}\left(x_{k}, t_{k}\right)=\min _{1 \leq i \leq n} R_{i \bar{i}}\left(x_{k}, t_{k}\right) .
$$

By the work of B.-L. Chen, X.-P. Zhu and the first author [9] (or by Perelman, see [25]), we know that the diameter and the curvature tensor of $g_{i \bar{j}}(t)$ are uniformly bounded. Also injectivity radius is uniformly bounded by Perelman's noncollapsing theorem [21]. Thus we can apply Hamilton's compactness theorem in [16] so that a subsequence of the compact marked solutions $\left\{\left(M^{n}, J, g_{i \bar{j}}\left(t_{k}+t\right), x_{k}, E^{k}\right)\right\}$ converges in $C^{\infty}$ norm, in the Cheeger-Gromov sense, to a compact marked solution $\left(M, \tilde{J}, \tilde{g}_{i \bar{j}}(t), \tilde{x}, \tilde{E}\right)$ to $(1.1)$ with nonnegative bisectional curvature $\tilde{R}_{i \bar{i} j \bar{j}} \geq 0$ and

$$
\tilde{R}_{1 \overline{1}}(\tilde{x}, 0)=\lim _{k \rightarrow \infty} R_{1 \overline{1}}\left(x_{k}, t_{k}\right)=0 .
$$

Here $\tilde{E}$ is a unitary frame at the marked point $\tilde{x}$ at $t=0$, and $\tilde{J}$ is a complex structure on $M^{n}$, possibly different from $J$. Moreover, we know that $\left(M, \tilde{J}, \tilde{g}_{i \bar{j}}(t)\right)$ is a gradient shrinking Kähler-Ricci soliton.

Now we use the following result of H.-L. Gu [12] which is proved by a strong maximum principle type of argument.

Lemma 2.1. Given any Kähler metric $h_{i \bar{j}}$ with nonnegative bisectional curvature on a compact, irreducible, simply connected Kähler manifold $N^{n}$. Then, under the normalized Kähler-Ricci flow (1.1), the bisectional curvature becomes positive everywhere after a short time, unless $\left(N^{n}, h_{i \bar{j}}\right)$ is isometrically biholomorphic to a Hermitian symmetric space of rank $\geq 2$.

We know that our manifold $M^{n}$ is simply connected (cf. [18]). Also, by a theorem of Bishop and Goldberg [3] (see also Theorem 4, [4]), $b_{2}\left(M^{n}\right)=1$. Hence, $M^{n}$ is irreducible. On the other hand, since $(M, \tilde{g})$ is a shrinking soliton with nonnegative bisectional curvature, (2.3) tells us that for each $t \in[0, \infty)$, the bisectional curvature $\tilde{R}_{i \bar{i} j \bar{j}}(t)$ vanishes somewhere at some point on $M^{n}$ at $t$. Therefore, Lemma 2.1 implies that $\left(M^{n}, \tilde{J}, \tilde{g}\right)$ is isometrically biholomorphic to a Hermitian symmetric space of rank $\geq 2$. In particular, $\tilde{g}$ is Kähler-Einstein, i.e., $\tilde{R}_{i \bar{j}}=\tilde{g}_{i \bar{j}}$. However this leads to a contradiction because $\tilde{g}$ being Kähler-Einstein in turn implies that

$$
\left\|R_{i \bar{j}}-g_{i \bar{j}}\right\|_{C^{0}}\left(t_{k}\right) \rightarrow 0,
$$

contradicting (2.2). This finishes the proof of the Claim.

On the other hand, by a direct computation of the evolution of

$$
S_{i \bar{j} k \bar{l}}(t)=R_{i \bar{j} k \bar{l}}(t)-c\left[g_{i \bar{j}} R_{k \bar{l}}+R_{i \bar{j}} g_{k \bar{l}}+g_{i \bar{l}} R_{k \bar{j}}+R_{i \bar{l}} g_{k \bar{j}}\right](t)
$$


for some sufficiently small positive constant $c>0$ and applying the maximum principle, Chen-Sun-Tian [10] (see Lemma 6 in [10]) showed that if the Ricci curvature of $g_{i \bar{j}}(t)$ has a uniform positive lower bound then the bisectional curvature of $g_{i \bar{j}}(t)$ has a uniform positive lower bound. Thus Theorem 1.1 follows.

In [24], Phong and Sturm first considered the smallest positive eigenvalue $\lambda$ of the operator $L=-g^{i \bar{j}} \nabla_{i} \nabla_{\bar{j}}$ acting on smooth $(1,0)$ vector fields. It has been demonstrated in [22] and $[23,24]$ that a uniform positive lower bound on $\lambda$ plays an important role in showing the convergence of the Kähler-Ricci flow.

Combining Theorem 1.1 above with Lemma 6 and Lemma 2 in [22], we obtain a uniform positive lower bound on $\lambda$ for solutions to the Kähler-Ricci flow (1.1) with positive bisectional curvature.

Corollary 2.1. Under the same assumption as in Theorem 1.1, there exists a positive constant $C_{2}>0$ such that the smallest positive eigenvalue $\lambda$ satisfies

$$
\lambda \geq C_{2}
$$

for all $t \geq 0$.

Remark 2.1. Under the additional assumption that the Mabuchi K-energy is bounded from below, both Theorem 1.1 and Corollary 2.1 were proved in [22].

Now we are ready to prove Theorem 1.2.

Proof of Theorem 1.2. Let $\left(M^{n}, g_{0}\right)$ be a compact Kähler manifold with positive bisectional curvature. Then, by the work of the first author [8], we know that the solution $g_{i \bar{j}}(t)$ to the normalized Kähler-Ricci flow (1.1) with initial metric $g_{0}$ exists for all time $0 \leq t<\infty$. Moreover, it follows from Bando [1] (for $n=3$ ) and Mok [19] (for $n \geq 3$ ) that $g_{i \bar{j}}(t)$ has positive bisectional curvature for all $t \geq 0$.

We will need the following result from [22],

Lemma 2.2. (Phong-Song-Sturm-Weinkove [22]) Suppose we have a nonsingular solution to the normalized Kähler-Ricci flow (1.1) on a compact Kähler manifold with positive first Chern class. Assume that the Futaki invariant is zero and the smallest positive eigenvalue $\lambda$ of the operator $L$ has a uniform positive lower bound. Then the solution converges to a Kähler-Einstein metric exponentially fast in $C^{\infty}$-norm.

Now our solution $g_{i \bar{j}}(t)$ is nonsingular by Cao-Chen-Zhu [9] (or Perelman, see [25]), and Futaki invariant is zero by assumption. Moreover, by Corollary 2.1, the smallest positive eigenvalue $\lambda$ of the operator $L=-g^{i \bar{j}} \nabla_{i} \nabla_{\bar{j}}$ has uniform positive lower bound. Thus it follows from Lemma 2.2 that $g_{i \bar{j}}(t)$ converges exponentially fast in $C^{\infty}$ to a Kähler-Einstein metric $\tilde{g}$. Also, by Theorem 1.1, $\tilde{g}$ has positive bisectional curvature. Therefore, by a theorem of M. Berger [2] (see also Theorem 5, [4]), $\tilde{g}$ is of constant holomorphice sectional curvature. The proof of Theorem 1.2 is completed.

\section{Acknowledgements}

We would like to thank Richard Hamilton, D.H. Phong, Jacob Sturm, Xiaofeng Sun and Valentino Tosatti for their interest in our work. 


\section{References}

[1] S. Bando, On the classification of three-dimensional compact Kähler, J. Diff. Geom. 19 (1984), no. 2, 283-297.

[2] M. Berger, Sur les variétés d'Einstein compactes, Comptes Rendus de la IIIe Réunion du Groupement des Mathématiciens d'Expression Latine (1965) 35-55.

[3] R. Bishop and S. I. Goldberg, On the second cohomology group of a Kähler manifold of positive curvature, Proc. Amer. Math. Soc. 16 (1965) 119-122.

[4] — Holomorphic bisectional curvature, J. Diff. Geom. (1967), no. 3, 225-233.

[5] C. Böhm and B. Wilking, Manifolds with positive curvature operator are space forms, Ann. of Math. 167 (2008) 1079-1097.

[6] S. Brendle and R. Schoen, Classification of manifolds with weakly 1/4-pinched curvatures, Acta Math. 200 (2008) 1-13.

[7] — Manifolds with 1/4-pinched Curvature are Space Forms, J. Amer. Math. Soc. 22 (2009), no. 1, 287-307.

[8] H.-D. Cao, Deformation of Kähler Metrics to Kähler-Einstein Metrics on compact Kähler manifolds, Invent. Math. 81 (1985), no. 2, 359-372.

[9] H.-D. Cao, B.-L. Chen, and X.-P. Zhu, Ricci flow on compact Kähler manifolds of positive bisectional curvature, C. R. Math. Acad. Sci. Paris 337 (2003), no. 12, 781-784.

[10] X. Chen, S. Sun, and G. Tian, A note on Kähler-Ricci soliton, arXiv:0806.2848 (2009)

[11] B. Chow, The Ricci flow on the 2-sphere, J. Diff. Geom. 33 (1991) 325-334.

[12] H.-L. Gu, A new proof of Mok's generalized Frankel conjecture theorem, Proc. Amer. Math. Soc. 137 (2009), no. 3, 1063-1068.

[13] R. S. Hamilton, Three manifolds with positive Ricci curvature, J. Diff. Geom. 17 (1982) 255-306.

[14] —, Four-manifolds with positive curvature operator, J. Diff. Geom. 24 (1986) 153-179.

[15] - The Ricci flow on surfaces, in Mathematics and general relativity, Contemp. Math., 17, 237-262, Amer. Math. Soc., Providence, RI (1988).

[16] — A compactness property for the Ricci flow, Amer. J. Math. 117 (1995) 545-572.

[17] - The formation of singularities in the Ricci flow, in Surveys in Differential Geometry, Vol. 2, 7-136, International Press, Combridge, MA (1995).

[18] S. Kobayashi, On compact Kähler manifolds with positive definite Ricci tensor, Ann. of Math. (2) 74 (1961) 570-574.

[19] N. Mok, The uniformization theorem for compact Kähler manifolds of nonnegative holomorphic bisectional curvature, J. Diff. Geom. 27 (1988) 179-214.

[20] S. Mori, Projective manifolds with ample tangent bundles, Ann. of Math., (2) 110 (1979), no. 3, 593-606.

[21] G. Perelman, The entropy formula for the Ricci flow and its geoemtric applicaitions, arxiv: math.DG/0211159 (2002)

[22] D. Phong, J. Song, J. Sturm, and B. Weinkove, The Kähler-Ricci flow with positive bisectional curvature, Invent. Math. 173 (2008), no. 3, 651-665.

[23] - The Kähler-Ricci flow and the $\bar{\partial}$ operator on vector fields, J. Diff. Geom. 81 (2009), no. 3, 631-647.

[24] D. Phong and J. Sturm, On stability and the convergence of the Kähler-Ricci flow, J. Diff. Geom. 72 (2006), no. 1, 149-168.

[25] N. Sesum and G. Tian, Bounding scalar curvature and diameter along the Kähler Ricci flow (after Perelman), J. Inst. Math. Jussieu 7 (2008), no. 3, 575-587.

[26] Y.-T. Siu and S.-T. Yau, Compact Kähler manifolds of positive bisectional curvature, Invent. Math. 59 (1980), no. 2, 189-204.

[27] F. Zheng, Complex differential geometry, AMS/IP Studies in Advanced Mathematics, 18, American Mathematical Society, Providence, RI, International Press, Boston, MA (2000).

Department of Mathematics, Lehigh University, Bethlehem, PA 18015

E-mail address: huc2@lehigh.edu

Department of Mathematics, Lehigh University, Bethlehem, PA 18015

E-mail address: mez206@lehigh.edu 